\title{
Context Expansion with Global Keywords for a Conceptual Density-Based WSD
}

\author{
Davide Buscaldi ${ }^{1}$, Paolo Rosso ${ }^{2}$, and Manuel Montes y Gómez ${ }^{3,2}$ \\ 1 Dipartimento di Informatica e Scienze dell'Informazione (DISI), \\ Università di Genova, Italy \\ buscaldi@disi.unige.it \\ 2 Dpto. de Sistemas Informáticos y Computación (DSIC), \\ Universidad Politecnica de Valencia, Spain \\ \{prosso,mmontes\}@dsic.upv.es \\ 3 Lab. de Tecnologías del Lenguaje \\ Instituto Nacional de Astrofísica, Optica y Electrónica, Mexico \\ mmontesg@inaoep.mx
}

\begin{abstract}
The resolution of the lexical ambiguity, which is commonly referred to as Word Sense Disambiguation, is still an open problem in the field of Natural Language Processing. An approach to Word Sense Disambiguation based on Conceptual Density (a measure of the correlation between concepts) obtained good results with small context windows. This paper presents a method to integrate global knowledge, expressed as global keywords, in this approach. Global keywords are extracted from documents using a model based on term frequency and distribution. Preliminary results show that a slight improvement in recall can be obtained over the base system.
\end{abstract}

\section{Introduction}

The resolution of lexical ambiguity that appears when a given word in a context has several different meanings is commonly referred as Word Sense Disambiguation (WSD). Supervised approaches to WSD usually perform better than unsupervised ones. However, such approaches are afflicted by the lack of large, semantically annotated corpora. The unsupervised approach to WSD based on Conceptual Density and the frequency of WordNet senses [4] is an unsupervised approach which obtained good results, in terms of precision, for the disambiguation of nouns over SemCor ( $81.55 \%$ with a context window of only two nouns, compared with the MFU-baseline of $75.55 \%$ ), and in the Senseval-3 all-words task $(73.40 \%$, compared with the MFU-baseline of $69.08 \%)$ as the CIAOSENSO2 system [2].

Our approach obtained the above results with a context window of only two nouns, one before and one after the noun to disambiguate, exploiting the relationship existing between adjacent words. The obtained results [4] show that a larger context deteriorates the performance of the approach. We suppose that such decrease is due to the fact that distant words have little or no meaning 
for the disambiguation of a given word. The only relationship which may exist between two distant words in the same document is that they are related to the content of the document itself.

In order to introduce this information into our approach we needed to select the most representative words in a document, and adding them to the context of the word to disambiguate. The selected model for extracting document keywords was based on term frequency and distribution as presented in [3].

\section{The CD-based Approach}

Conceptual Density (CD) is a measure of the correlation among the sense of a given word and its context. Our approach carries out the noun sense disambiguation by means of a formula [4], derived from the original Conceptual Density described in [1].

Due to the granularity of the version 2.0 of WordNet, we consider only the relevant part of the subhierarchy determined by the synset paths (from the synset at the top of subhierarchies to an ending node) of the senses of both the noun to be disambiguated and its context, and not the portion of subhierarchy constituted by the synsets that do not belong to the synset paths. Moreover, information about word frequency contained in WordNet was also taken into consideration. A more detailed description of the method could be found in [2].

\section{Extraction of Global Keywords}

Document keywords appear usually in very different locations in the document. The Information Retrieval (IR) model proposed by [3] allows to use distribution characteristics of words to determine keywords, by computing their standard deviation. The standard deviation for the $i$-th word in a document is computed as:

$$
s_{i}^{2}=\frac{1}{\left(f_{i}-1\right)} \sum_{j}\left(l_{i j}-m_{j}\right)^{2}
$$

where $f_{i}$ is the frequency of the $i$-th word, $l_{i j}$ is the $j$-th position of the word in document, and $m_{j}$ is the mean of relative location $j$. Thereafter, we can extract document keywords, having great frequency and standard deviation, that is, wide distribution over the text.

We applied this IR model to the three documents which are part of the Senseval-3 all-words corpus, obtaining the global keywords as showed in the Table 1 .

Document 1 is a part of a novel, document 2 is a newspaper article about presidential elections, while document 3 is a collection of excerpts from a bulletin board. It is noteworthy how representative are the global keywords extracted from document 2 . 


\begin{tabular}{|l|l|r|r|r|}
\hline Document & keywords & frequency & positions & deviation \\
\hline doc1 & guy & 5 & $65,229,648,1658,1875$ & 330.8 \\
& course & 4 & $124,990,1207,1994$ & 332.9 \\
& something & 4 & $202,1011,1127,1907$ & 302.1 \\
& accident & 4 & $776,1193,1969,1999$ & 260.6 \\
\hline doc2 & level & 4 & $33,1271,1278,1344$ & 274.2 \\
& ticket & 4 & $35,490,789,1258$ & 222.6 \\
& gop & 5 & $6,126,431,951,1232$ & 211.3 \\
& pattern & 4 & $155,498,891,1266$ & 208.4 \\
& election & 5 & $51,113,510,666,1200$ & 186.4 \\
\hline doc3 & berkeley & 3 & $278,356,1405$ & 296.7 \\
& bay & 3 & $11,96,1105$ & 286.9 \\
& line & 3 & $59,454,1214$ & 276.7 \\
& phone & 3 & $58,723,1213$ & 273.3 \\
& book & 3 & $301,663,1283$ & 234.1 \\
& room & 3 & $306,662,1289$ & 234.6 \\
& night & 3 & $5,128,887$ & 225.2 \\
\hline
\end{tabular}

Table 1. Keywords extracted for each document in the Senseval-3 all-words corpus, sorted by standard deviation. Frequency is the total number of occurrences in the document, positions are the numbers identifying words' positions in the document, deviation is the standard deviation calculated over the document.

\section{Experimental Results}

The Global Keywords (GK) extracted were added to the context of each word, taking them into account for the computation of Conceptual Density. Table 2 shows the obtained results, compared with those obtained with the CIAOSENSO2 system at Senseval-3 [2] and the Most Frequent Sense (MFS) heuristic.

We obtained a slight improvement in Recall $(1.7 \%)$ and Coverage $(\sim 3 \%)$, but there was a $\sim 1 \%$ loss in precision. In order to obtain better results, we decided to add to the context only two words for each document. The two words were selected on the basis of the following criteria:

1. Polysemy (i.e., those having fewer senses);

2. Depth in the WordNet hierarchy (i.e., the words whose synsets' average depth is the greatest);

3. Specificity (i.e., the words whose synsets' averaged number of hyponyms is smaller).

Table 2 compares the results which were obtained over the nouns of the English all-words Senseval-3 corpus using the CD approach standalone, together with the context expanded with the whole global information, as well as after filtering it depending on polysemy, averaged synsets depth, and averaged number of hyponyms characteristics. 


\begin{tabular}{|l|r|r|r|}
\hline & Precision & Recall & Coverage \\
\hline CIAOSENSO-2 & 0.743 & 0.497 & $66.9 \%$ \\
MFS & 0.691 & 0.691 & $100 \%$ \\
CD+GK & 0.734 & 0.508 & $69.2 \%$ \\
\hline CD+Less Polysemic & 0.729 & 0.506 & $69.3 \%$ \\
CD+Deepest & 0.731 & 0507 & $69.3 \%$ \\
CD+Most Specific & 0.730 & 0.507 & $69.4 \%$ \\
\hline
\end{tabular}

Table 2. Results obtained over the nouns in the Senseval-3 all-words corpus using context expanded with GK $(C D+G K)$, the CD approach (CIAOSENSO-2), the MFS heuristic, and filtering GK by their characteristics - polisemy (CD+Less Polysemic), averaged depth of synsets (CD+Deepest), and averaged number of hyponyms (CD+Most Specific).

\section{Conclusions and Further Work}

The number of the experiments and the size of the used corpus are too small to fully understand the impact of representative global information on WSD. However, it seems that a slight improvement in recall and coverage can be obtained without losing too much in precision. This has to be proved over a larger corpus, such as SemCor. Filtering global keywords depending on their polisemy, depth and hyponyms features extracted from WordNet did not prove to be helpful in WSD, even if we suppose that this can be exploited in other applications, such as IR, to improve the model based on frequency and distribution of words.

\section{Acknowledgments}

We would like to thank R2D2 CICYT (TIC2003-07158-C04-03), CONACyTMexico (43990A-1, U39957-Y) and ICT EU-India (ALA/95/23/2003/077-054) projects, as well as the Secretaría de Estado de Educación y Universidades de España, for partially supporting this work.

\section{References}

1. Agirre, E., Rigau, G.: A proposal for Word Sense Disambiguation using Conceptual Distance. Proc. of the Int. Conf. on Recent Advances in NLP (RANLP'95). 1995.

2. Buscaldi, D., Rosso, P., Masulli, F.: The upv-unige-CIAOSENSO WSD System. Senseval-3 Workshop, Association for Computational Linguistics (ACL-04). Barcelona, Spain (2004).

3. Lee, J., Baik, D..: A Model for Extracting Keywords of Document Using Term Frequency and Distribution Lecture Notes in Computer Science, Vol. 2588. SpringerVerlag (2004)

4. Rosso, P., Masulli, F., Buscaldi, D., Pla, F., Molina, A.: Automatic Noun Disambiguation. Lecture Notes in Computer Science, Vol. 2588. Springer-Verlag (2003) 273-276. 